\title{
TOPOLOGICAL INVARIANCE OF IDEALS IN MOBS ${ }^{1}$
}

\section{A. D. WALLACE}

A $m o b$ is a Hausdorff space together with a continuous associative multiplication. In all that follows $S$ will be a compact mob. A set $T \subset S$ is a left ideal if $T \neq \square$ and if $S T \subset T$. It is clear how to define right ideal and (two-sided) ideal. Numakura [7] has shown that $S$ contains minimal ideals of all three sorts and these are closed sets. We let $K$ be the minimal ideal of $S$. Improving some results of [11], we show among other things that, with additional assumptions on $S$, it is possible to give a completely topological definition of $K$. It will be seen also that if $N$ is a sufficiently "large" subgroup of $S$, then the cohomology structure of $S$ is the same as that of $N$. This will be done by showing that $N=K$. From this it follows that $N$ is a homomorphic retract of $S$. But $N$ need not be a deformation retract of $S$, see [3].

The Alexander-Kolmogoroff cohomology group of the space $X$ with coefficient group $G$ will be denoted by $H^{n}(X, G)$, Spanier [8]. We sometimes write $H^{n}(X)$ for $H^{n}(X, G)$. It is possible to define a dimension function (Haskell Cohen [4]) by letting $c d(X, G) \leqq n$ if the natural homomorphism $H^{n}(X, G)$ into $H^{n}(A, G)$ is onto for each closed $A \subset X$. If $X$ is compact Hausdorff then $c d(X$, integers) is the covering dimension, [1] and [5]. Cohen [4] showed that $c d(X, G)$ cannot exceed the covering dimension for a compact $X$. If $h \in H^{n}(X)$, then $h \mid A$ will denote the image of $h$ in $H^{n}(A)$ under the natural homomorphism, $A \subset X$. A continuum is a compact connected Hausdorff space.

Lemma. Let $A$ be a compact set in $S$ and let $Z$ be a continuum in $S$ such that $c d(Z A, G) \leqq n$. Let $p, q \in Z$ and define $f: A \rightarrow q A$ by $f(x)=q x$. If $h \in H^{n}(q A, G)$ and if $h \mid(p A \cap q A)=0$, then $f^{*}(h)=0$. If also $q^{2}=q$ and $q A \subset A$, then $h=0$.

Proof. In the Mayer-Vietoris sequence $\left[6\right.$, p. 43; 10] $H^{n}(q A \cup p A)$ $\rightarrow H^{n}(q A) \times H^{n}(p A) \rightarrow H^{n}(q A \cap p A)$, the element $(h, 0)$ of the middle term goes into the zero of the last term, so that $(h, 0)$ is the image of an element $h_{1} \in H^{n}(q A \cup p A)$. Since $c d(Z A) \leqq n$ and since $q A \cup_{p} A$ is closed in $Z A$, we have $h_{1}=h_{2} \mid(q A \cup p A)$ for some $h_{2} \in H^{n}(Z A)$. Define $g: A \rightarrow p A$ by $g(x)=p x$ and $g_{0}, f_{0}: A \rightarrow Z A$ by $g_{0}(x)=g(x), f_{0}(x)=f(x)$. By $[11$, p. 47$]$, we know that $f_{0}^{*}=g_{0}^{*}$. Now

Presented to the Society, September 3, 1954; received by the editors April 12, 1954.

1 This work was done under Contract N7-onr-434, Task Order III, Navy Department, Office of Naval Research. 


$$
f_{0}^{*}\left(h_{2}\right)=f^{*}\left(h_{2} \mid q A\right)=f^{*}(h)
$$

and

$$
g_{0}^{*}\left(h_{2}\right)=g^{*}\left(h_{2} \mid p A\right)=g^{*}(0)=0 .
$$

Hence $f^{*}(h)=0$. If $q^{2}=q$ and $q A \subset A$, then $f$ is a retraction. Thus $f^{*}$ is an isomorphism so that $f^{*}(h)=0$ gives $h=0$.

If $X$ is compact Hausdorff and if $h \in H^{p}(X, G)$ is not zero, then there is a closed set $F \subset X$ such that $h \mid F \neq 0$, but $h \mid F_{0}=0$ for any closed proper subset $F_{0}$ of $F$. We term $F$ a floor for $h$, see [9].

REMARK. If $S$ is compact, if $A$ is closed in $S$, and if $t_{0} \in S$, then $A \subset t_{0} A$ implies $A=t_{0} A$ and also $A=e A$ for some $e \in S$, with $e^{2}=e$ $[12$, p. 24].

THEOREM. Let $S$ be a compact connected mob with $c d(S, G) \leqq n$ and let $N$ be a closed set in $S$ with $H^{n}(N, G) \neq 0$ and with $N \subset t_{1} N$ for some $t_{1} \in S$. Then $K$, the minimal ideal of $S$, is also a minimal right ideal and $K$ contains every floor for every nonzero $h \in H^{n}(N, G)$ and each such floor is a left ideal of $S$. If also $N \subset N t_{2}$ for some $t_{2} \in S$, then $K$ is a group and is the unique floor for each nonzero $h$ in $H^{n}(N, G)$.

Proof. Let $h$ be a nonzero element of $H^{n}(N)$ and let $A$ be a floor for $h$. Since $N \subset t_{1} N$, we have $N=e N$ for some $e \in S$ with $e^{2}=e$. Hence $A=e A$ because $A \subset N=e N$. Let $h_{0}=h \mid A$ and let $t \in S$. Now $A$ is a floor for $h_{0}$, so that if $A$ is not contained in $t A$ then $A \cap t A$ is a proper subset of $A$, and thus $h_{0} \mid(e A \cap t A)=0$, recalling that $A=e A$. By the lemma, $h_{0}=0$ contrary to the fact that $A$ is a floor for $h$. Thus $A \subset t A$ and hence $A=t A$. Take any $f \in K$ with $f^{2}=f$, see $[2$, p. 525]. Then $A=f A \subset f S \subset K$ and $f S$ is a minimal right ideal. Now all minimal right ideals are obtainable as $f S$ for some $f \in K$ with $f^{2}=f$ and because $K$ is the union of all minimal right ideals, we see that $K=f S$ for any such $f$ and so $K$ is a minimal right ideal, see [2]. It is clear that $A$ is a left ideal. If also $N \subset N t_{2}$, then by left-right duality we have $A=K$.

Hence we see that $K$ is a group and if $e$ is the unit of $K$, then $x e=e x$ for each $x \in S$ and $x \rightarrow x e$ is a retracting homomorphism, see [3]. It also follows that (taking $S=N$ ) $K$ can be defined as the unique floor for any nonzero $h \in H^{n}(S, G)$, so that $K$ is a topological invariant of $S$ in the following sense: Let $S$ be a clan (= compact connected mob with (two-sided) unit), let $c d(S, G) \leqq n$, and let $H^{n}(S, G) \neq 0$. If $T$ is a mob with unit and if $f$ is a homeomorphism of $S$ on to $T$, then $f$ takes the minimal ideal of $S$ onto the minimal ideal of $T$. The hypothesis $H^{n}(S, G) \neq 0$ is essential to this result.

Corollary. Let $S$ be a clan and for some coefficient group $G_{0}$, let 
$c d\left(S, G_{0}\right) \leqq n$ and let $N$ be a closed subgroup of $S$ with $H^{n}\left(N, G_{0}\right) \neq 0$. Then $K=N$ and hence $N$ is a homomorphic retract of $S$ and $H^{p}(N, G)$ is naturally isomorphic with $H^{p}(S, G)$ for any $p \geqq 0$ and any coefficient group $G$.

Proof. By the theorem we know that $K \subset N$ so that $K=N$ because $N$ is a group. That $H^{p}(S, G) \approx H^{p}(K, G)$ is known, [11, p. 48].

\section{BIBLIOGRAPHY}

1. P. Alexandroff, On the dimension of normal spaces, Proc. Roy. Soc. London Ser. A vol. 189 (1947) pp. 11-39.

2. A. H. Clifford, Semigroups containing minimal ideals, Amer. J. Math. vol. 70 (1948) pp. 521-526.

3. A. H. Clifford and D. D. Miller, Semigroups having zeroid elements, Amer. J. Math. vol. 70 (1948) pp. 117-125.

4. H. Cohen, A cohomological definition of dimension for locally compact Hausdorff spaces, Duke Math. J. vol. 21 (1954) pp. 209-224.

5. H. Dowker, Mapping theorems for non-compact spaces, Amer. J. Math. vol. 69 (1947) pp. 200-242.

6. S. Eilenberg and N. E. Steenrod, Foundations of algebraic topology, Princeton, 1952.

7. K. Numakura, On bicompact semigroups, Math. J. of Okayama Univ. vol. 1 (1952) pp. 99-108.

8. E. Spanier, Cohomology theory for general spaces, Ann. of Math. vol. 49 (1948) pp. $407-427$.

9. A. D. Wallace, Outline for algebraic topology, Tulane University, 1949-1952.

10. — The map excision theorem, Duke Math. J. vol. 19 (1952) pp. 177-182.

11. - Cohomology, dimension and mobs, Summa Brasil. Math. vol. 3 (1953) pp. $43-54$.

12. - Inverses in Euclidean mobs, Math. J. of Okayama Univ. vol. 3 (1953) pp. 23-28.

The Tulane University of Louisiana 